Research Article

\title{
Some Criteria for Class Numbers to Be Non-One
}

\author{
Ahmad Issa (iD) and Hasan Sankari \\ Department of Mathematics, Tishreen University, Lattakia, Syria \\ Correspondence should be addressed to Ahmad Issa; ahmad.issa@tishreen.edu.sy \\ Received 3 June 2020; Accepted 14 October 2020; Published 17 November 2020 \\ Academic Editor: Li Guo \\ Copyright (C) 2020 Ahmad Issa and Hasan Sankari. This is an open access article distributed under the Creative Commons \\ Attribution License, which permits unrestricted use, distribution, and reproduction in any medium, provided the original work is \\ properly cited. \\ Let $d$ be a positive integer which is not a perfect square and $n$ be any nonzero fixed integer. Then, the equation $x^{2}-\mathrm{d} y^{2}=n$ is \\ known as the general Pell equation. In this paper, we give some criteria for class numbers of certain real quadratic fields to be \\ greater than one, depending on the solvability of the general Pell equation, ideals in quadratic orders, and the period length of the \\ simple continued fraction expansions of $\sqrt{d}$.
}

\section{Introduction}

Let $d$ be a positive square-free integer, $k=\mathbb{Q}(\sqrt{d})$ be a real quadratic field, and $h(d)$ be the class number of this field.

The class number problem of quadratic fields is one of the most intriguing unsolved problems in the algebraic number theory, and it has been the object of attention for many years of researchers.

In [1], Gauss had conjectured that there exist exactly nine imaginary quadratic fields of class number 1 . Later, this was solved after diverse works of Stark, Heegner, and Baker. And more, he conjectured that there exist infinitely many real quadratic fields of the form $\mathbb{Q}(\sqrt{p}), p \equiv 1(\bmod 4)$, of class number 1.

Many fruitful research studies have been done in this direction. Biro' (see $[2,3]$ ) proved in 2003 two important results: Yokoi's conjecture which asserts that $h\left(m^{2}+4\right)=1$ only for six values of $m=1,3,5,7,13,17$ and Chowla's conjecture which says that $h\left(4 m^{2}+1\right)=1$ only for six values of $m=1,2,3,5,7,13$.

Mollin [4] considered $m^{2}+1$ and proved that if $m \neq 1$, $2 q$, where $q$ is a prime, then $h\left(m^{2}+1\right)>1$. In 2007, Byeon et al. proved (see [5]) Mollin's conjecture which says that $h\left(m^{2}-4\right)>1$ whenever $m>21$. Numerous recent research studies have shown that the class number for a given family of quadratic fields is greater than one (see [6-14]).
In this work, we give some possible generators of the principal primitive ideal by special cases of general Pell equations' solutions. Since it is not always easy to check whether the class number for a given family of fields is greater than one or equals one, we give some results about that.

\section{Preliminaries}

Herein, we will be concerned with the simple continued fraction expansions of $\sqrt{d}$, where $d$ is a positive integer that is not a perfect square. We denote this expansion by

$$
\alpha=\sqrt{d}=\left\langle q_{0} ; \overline{q_{1}, q_{2}, \ldots, q_{\ell-1}, 2 q_{0}}\right\rangle,
$$

where $\ell=\ell(\sqrt{d})$ is the period length, $q_{0}=\lfloor\sqrt{d}\rfloor$ (the floor of $\sqrt{d})$, and $q_{1}, q_{2}, \ldots, q_{\ell-1}$ is a palindrome.

The $k$-th convergent of $\alpha$ for $k \geq 0$ is given by

$$
\frac{A_{k}}{B_{k}}=\left\langle q_{0} ; q_{1}, q_{2}, \ldots, q_{k}\right\rangle
$$

where

$$
\begin{aligned}
& A_{k}=q_{k} A_{k-1}+A_{k-2}, \\
& B_{k}=q_{k} B_{k-1}+B_{k-2},
\end{aligned}
$$


with $A_{-2}=0, A_{-1}=1, B_{-2}=1$, and $B_{-1}=0$. The complete quotients are given by $\left(P_{k}+\sqrt{d}\right) / Q_{k}$, where $P_{0}=0, Q_{0}=1$, and for $k \geq 1$,

$$
\begin{aligned}
P_{k+1} & =q_{k} Q_{k}-P_{k}, \\
q_{k} & =\left\lfloor\frac{P_{k}+\sqrt{d}}{Q_{k}}\right\rfloor,
\end{aligned}
$$

and $d=P_{k+1}^{2}+Q_{k} Q_{k+1}$. We will also need the following fact:

$$
A_{k-1}^{2}-\mathrm{d} B_{k-1}^{2}=(-1)^{k} Q_{k} .
$$

Also, when $\ell=\ell(\sqrt{d})$ is even, $Q_{(\ell / 2)}$ is called the central norm (for more information, see $[15,16]$ ).

Now, we will introduce some basic about quadratic order in the quadratic number field. A nonsquare integer $\Delta \in \mathbb{Z}$ is called a quadratic discriminant if $\Delta \equiv 0$ or $1(\bmod 4)$. The quadratic discriminant $\Delta$ is called a fundamental discriminant if it admits no factorization $\Delta=\Delta_{1} m^{2}$ such that $\Delta_{1}$ is a quadratic discriminant and $m \in \mathbb{N}_{\geq 2}$. In addition, $\Delta$ is a fundamental discriminant if and only if either $\Delta$ is squarefree and $\Delta \equiv 1(\bmod 4)$ or $\Delta=4 d$ for some square-free integer $d$ such that $d \equiv 2$ or $3(\bmod 4)$.

Let $\Delta$ be a quadratic discriminant, then the order of the quadratic discriminant $\Delta$ is

$$
\sigma_{\Delta}=\left\{\frac{u+v \sqrt{\Delta}}{2} \mid u, v \in \mathbb{Z}, u \equiv v \Delta(\bmod 2)\right\},
$$

and if $\Delta$ is a fundamental discriminant, we have $\mathcal{O}_{\Delta}=\mathcal{O}_{k}$, where $\mathscr{O}_{k}$ is the ring of integers of $k=\mathbb{Q}(\sqrt{\Delta})$. The unit group $\mathcal{O}_{\Delta}^{\times}$of $\mathcal{O}_{\Delta}$ is given by $\mathcal{O}_{\Delta}^{\times}=\left\{\varepsilon \in \mathcal{O}_{\Delta}|| N(\varepsilon) \mid=1\right\}=$ $\left\{(u+v \sqrt{\Delta}) / 2|u, v \in \mathbb{Z},| u^{2}-\Delta v^{2} \mid=4\right\}$ (see [15], Theorem 5.2.1, p. 122).

Now, we investigate ideals. Let $\Delta$ be a quadratic discriminant. An ideal $I \subset \mathcal{O}_{\Delta}$ is called $\mathcal{O}_{\Delta}$-primitive if $e^{-1} I \nsubseteq$ $\mathcal{O}_{\Delta}$, for all $e \in \mathbb{N}_{\geq 2}$. For a nonzero ideal $I$ of $\mathcal{O}_{\Delta}$, we call $N(I)=\left(\mathcal{O}_{\Delta}: I\right)$ the absolute norm of $I$. If $\alpha$ is a nonzero of $\mathcal{O}_{\Delta}$, then $N(\langle\alpha\rangle)=|N(\alpha)|$ (see [15], Definition 5.4.1, p. 132).

\section{Main Results}

In this section, we will prove our main results. As a start, we record the following theorem.

\section{Theorem 1. Let $\Delta \in \mathbb{N}$ be a fundamental discriminant.}

(1) Suppose that $\Delta=4 d, c \in\{1,2\}$ if $d \equiv 3(\bmod 4)$, and $c=1$ if $d \equiv 2(\bmod 4) d=a b$, where $a, b \in \mathbb{N}$, and set

$$
I= \begin{cases}\langle a, \sqrt{d}\rangle, & \text { if } c=1, \\ \langle 2 a, a+\sqrt{d}\rangle, & \text { if } c=2 .\end{cases}
$$

(a) $I$ is an $\mathcal{O}_{k}$-primitive ideal of $\mathcal{O}_{k}$ satisfying $N(I)=c a$. In addition, the following assertions are equivalent:

(1) $I$ is a principal ideal of $\mathcal{O}_{k}$
(2) There exist $x, y \in \mathbb{Z}$ such that $\left|x^{2}-d y^{2}\right|=c a$

(b) Let $x, y \in \mathbb{Z}$ be such that $\left|x^{2}-d y^{2}\right|=c a$. Then, $I=\langle x+y \sqrt{d}\rangle$.

(2) Suppose that $\Delta \equiv 1(\bmod 4)$ and $\Delta=a b$, where $a, b \in \mathbb{N}$, and set $I=\langle a,(a+\sqrt{\Delta}) / 2\rangle$ :

(a) $I$ is an $\mathcal{O}_{k}$-primitive ideal of $\mathcal{O}_{k}$ satisfying $N(I)=a$. In addition, the following assertions are equivalent:

(1) $I$ is a principal ideal of $\mathcal{O}_{k}$

(2) There exist $x, y \in \mathbb{Z}$ such that $\left|x^{2}-\Delta y^{2}\right|=4 a$

(b) Let $x, y \in \mathbb{Z}$ be such that $\left|x^{2}-\Delta y^{2}\right|=4 a$. Then, $I=\langle(x+y \sqrt{\Delta}) / 2\rangle$.

(3) Suppose that $\Delta \equiv 1(\bmod 8)$ and $\Delta=a b$, where $a, b \in \mathbb{N}$, and set $I=\langle a,(a+\sqrt{\Delta}) / 2\rangle$ :

(a) $I$ is an $\mathcal{O}_{k^{-}}$primitive ideal of $\mathcal{O}_{k}$ satisfying $N(I)=a$. In addition, the following assertions are equivalent:

(1) $I$ is a principal ideal of $\mathcal{O}_{k}$

(2) There exist $x, y \in \mathbb{Z}$ such that $\left|x^{2}-\Delta y^{2}\right|=a$

(b) Let $x, y \in \mathbb{Z}$ be such that $\left|x^{2}-\Delta y^{2}\right|=a$. Then, $I=\langle x+y \sqrt{\Delta}\rangle$.

Proof

(1) (a) By [17] (Theorem 7.1.5, p. 144), we find that $I \subset \mathcal{O}_{k}$ is an ideal of $\mathcal{O}_{k}$ satisfying $N(I)=c a$. Let $e^{-1} I \subset \mathcal{O}_{k}$ with $e \in \mathbb{N}$ which implies $e=1$. Therefore, $I$ is an $\mathscr{O}_{k}$-primitive ideal of $\mathscr{O}_{k}$.

$(1) \Longrightarrow(2)$. Now, let $I$ be principal. Then, there exists $\alpha=x+y \sqrt{d} \in \mathcal{O}_{k}$, where $I=\langle\alpha\rangle$. Since $I$ is an $\mathcal{O}_{k}$-primitive ideal, gcd $(x, y)=1$.

If $c=1, I=\langle a, \sqrt{d}\rangle=\langle x+y \sqrt{d}\rangle$, then

$$
a=N(I)=|N(x+y \sqrt{d})|=\left|x^{2}-d y^{2}\right| .
$$

On the other hand,

If $c=2, I=\langle 2 a, a+\sqrt{d}\rangle=\langle x+y \sqrt{d}\rangle$, then

$$
2 a=N(I)=|N(x+y \sqrt{d})|=\left|x^{2}-\mathrm{d} y^{2}\right| .
$$

$(2) \Longrightarrow(1)$. Let $x, y \in \mathbb{Z}$ such that $\left|x^{2}-\mathrm{d} y^{2}\right|=c a$, which implies $x=a x_{1}$ for some $x_{1} \in \mathbb{Z}$.

If $c=1$, now we obtain $x+y \sqrt{d}=\left(a x_{1}+y \sqrt{d}\right) \in I$. Hence, $x+y \sqrt{d} \subset \mathrm{I}$, and equality holds, since

$$
\begin{aligned}
N(<x+y \sqrt{d}>) & =|N(x+y \sqrt{d})| \\
& =\left|x^{2}-\mathrm{d} y^{2}\right|=a=N(I) .
\end{aligned}
$$

On the other hand,

If $c=2$, which implies $\operatorname{gcd}(2, x y)=1$, then $\operatorname{gcd}\left(2, x_{1}\right)=1$ and $x_{1}-y=2 w$ for some $w \in \mathbb{Z}$. Now, we obtain $x+y \sqrt{d}=2 w a+y(a+\sqrt{d}) \in I$. Hence, $\langle x+y \sqrt{d}\rangle \subset I$, and equality holds, since 


$$
\begin{aligned}
N(<x+y \sqrt{d}>) & =|N(x+y \sqrt{d})| \\
& =\left|x^{2}-\mathrm{d} y^{2}\right|=2 a=N(I) .
\end{aligned}
$$

(b) By (2) $\Longrightarrow(1)$.

(2) (a) By [17] (Theorem 7.1.5, p. 144), we find that I $\subset \mathcal{O}_{k}$ is an ideal of $\mathscr{O}_{k}$ satisfying $N(I)=a$. Let $e^{-1} I \subset \mathcal{O}_{k}$ with $e \in \mathbb{N}$ which implies $e=1$. Therefore, $I$ is an $\mathscr{O}_{k}$-primitive ideal of $\mathscr{O}_{k}$.

(1) $\Longrightarrow(2)$. Let now $I$ be principal. Then, there exists $\alpha=(x+y \sqrt{\Delta}) / 2 \in \mathcal{O}_{k}$, where $I=\langle\alpha\rangle$. On the other hand, $a=N(I)=|N(\alpha)|=\left(\left|x^{2}-\Delta y^{2}\right| / 4\right)$, and therefore $\left|x^{2}-\Delta y^{2}\right|=4 a$.

(2) $\Longrightarrow(1)$. Let $x, y \in \mathbb{Z}$ such that $\left|x^{2}-\Delta y^{2}\right|=4 a$, which implies $2 \mid(x-y)$ and $x=a x_{1}$ for some $x_{1} \in \mathbb{Z}$. Now, we obtain $(x+y \sqrt{\Delta}) / 2=\left(a x_{1}+y \sqrt{\Delta}\right) / 2=a\left(x_{1}-y\right) / 2+$ $y(a+\sqrt{\Delta}) / 2 \in I$.

Hence, $(\langle x+y \sqrt{\Delta}\rangle / 2) \subset \mathrm{I}$, and equality holds, since

$$
N\left(\frac{\langle x+y \sqrt{\Delta}\rangle}{2}\right)=\left|N\left(\frac{(x+y \sqrt{\Delta})}{2}\right)\right|=a=N(I) .
$$

(b) By (2) $\Longrightarrow(1)$.

(3) (a) We note $I \subset \mathcal{O}_{k}$ is an $\mathcal{O}_{k}$-primitive ideal of $\mathscr{O}_{k}$ satisfying $N(I)=a$.

$(1) \Longrightarrow(2)$. Let now $I$ be principal. Then, there exist $u, v \in \mathbb{Z}$ such that $\left|u^{2}-\Delta v^{2}\right|=4 a$. Since $\Delta \equiv 1$ $(\bmod 8), \Delta$ is odd. But $4 a$ is even, so the equation $\mid u^{2}-$ $\Delta v^{2} \mid=4 a$ implies that $u$ and $v$ have the same parity. Let $u_{1}=(u-v) / 2, v_{1}=v$, hence $\mid u_{1}^{2}+u_{1} v_{1}+((1-\Delta) \mid$ 4) $v_{1}^{2}|=|\left(\left(4 u_{1}^{2}+4 u_{1} v_{1}+(1-\Delta) v_{1}^{2}\right) / 4\right) \mid=\left(\left|u^{2}-\Delta v^{2}\right| / 4\right)$ $=(4 a / 4)=a$. Since $a$ is odd and $((1-\Delta) / 4)$ is even, then $u_{1}$ is odd and $v_{1}$ is even. Let $m=\left(\left(2 u_{1}+v_{1}\right) / 2\right)$, $n=\left(v_{1} / 2\right)$, hence $\left|m^{2}-\Delta n^{2}\right|=\mid u_{1}^{2}+u_{1} v_{1}+((1-\Delta) \mid$ 4) $v_{1}^{2} \mid=a$. Let $x=m, y=n$, then there exist $x, y \in \mathbb{Z}$ such that $\left|x^{2}-\Delta y^{2}\right|=a$.

(2) $\Longrightarrow(1)$. Let $x, y \in \mathbb{Z}$ such that $\left|x^{2}-\Delta y^{2}\right|=a$. Then, $\left|(2 x)^{2}-\Delta(2 y)^{2}\right|=4 a$, which implies $2 x=a x_{1}$ for some $x_{1} \in \mathbb{Z}$. Now, we obtain $x+y \sqrt{\Delta}=$ $\left(a x_{1} / 2\right)+y \sqrt{\Delta}=a\left(\left(x_{1} / 2\right)-y\right)+2 y((a+\sqrt{\Delta}) / 2) \in I$. Hence, $\langle x+y \sqrt{\Delta}\rangle \subset I$, and equality holds, since

$N(<x+y \sqrt{\Delta}>)=|N((x+y \sqrt{\Delta}))|=a=N(I)$.

(b) By (2) $\Longrightarrow(1)$.
Now, we will be devoted to numerical examples illustrating Theorem 1.

Example 1. Let $\Delta=312=4 d ; d=78=3 \times 26=a b \equiv$ $2(\bmod 4)$. Since $(9,1)$ is the solution of $x^{2}-78 y^{2}=3$, then $\langle 3, \sqrt{d}\rangle=\langle 9+\sqrt{d}\rangle$.

Example 2. Let $\Delta=140=4 d ; d=35=5 \times 7=a b \equiv$ $3(\bmod 4)$. Since $(5,1)$ is the solution of $x^{2}-35 y^{2}=-10$, then $\langle 10,5+\sqrt{d}\rangle=\langle 5+\sqrt{d}\rangle$.

Example 3. Let $\Delta=805=5 \times 161=a b \equiv 1(\bmod 4)$. Since $(85,3)$ is the solution of $x^{2}-805 y^{2}=-20$, then $\langle 5,(5+$ $\sqrt{\Delta}) / 2\rangle=\langle(85+3 \sqrt{\Delta}) / 2\rangle$.

Example 4. Let $\Delta=129=3 \times 43=a b \equiv 1(\bmod 8)$. Since $(159,14)$ is the solution of $x^{2}-129 y^{2}=-3$, then $\langle 3,(3+$ $\sqrt{\Delta}) / 2\rangle=\langle 159+14 \sqrt{\Delta}\rangle$.

We need the following proposition in order to prove that $h\left((a m)^{2}+a\right)>1$.

Proposition 1. Let $\Delta \in \mathbb{N}$ be a quadratic discriminant, $\Delta=4 d$ or $\Delta=d \equiv 1(\bmod 8), d=(a m)^{2}+a$, where $a, m \in \mathbb{N}, m \geq 1, a>2$. Then, the equation $\left|x^{2}-d y^{2}\right|=p$ has no integer solutions for all primes $p$, where $a>p$.

Proof. We will provide the complete proof for the case $x^{2}-\mathrm{d} y^{2}=-p$, and the other case $x^{2}-\mathrm{d} y^{2}=p$ can be handled along the similar lines. Suppose this equation $x^{2}-$ $\mathrm{d} y^{2}=-p$ has integer solutions $(u, v)$, choose from these solutions $\left(u_{0}, v_{0}\right)$ with $u_{0} \geq 0, v_{0}>0$, and $v_{0}$ is the smallest. Let $x=u_{0}-v_{0} \sqrt{d} \in \mathcal{O}_{\Delta}$ and $\varepsilon=1+2 a m^{2}+2 m \sqrt{d} \in \mathcal{O}_{\Delta}^{\times}$. Then,

$$
N(\varepsilon x)=N(\varepsilon) N(x)=u_{0}^{2}-\mathrm{d} v_{0}^{2}=-p .
$$

Hence,

$$
\begin{aligned}
N\left(\left(1+2 a m^{2}+2 m \sqrt{d}\right)\left(u_{0}-v_{0} \sqrt{d}\right)\right) & =-p \\
\left(\left(1+2 a m^{2}\right) u_{0}-2 m v_{0} d\right)^{2}-d\left(2 m u_{0}-v_{0}\left(1+2 a m^{2}\right)\right)^{2} & =-p .
\end{aligned}
$$

By the definition of $v_{0}$, we find

$$
\left|2 m u_{0}-v_{0}\left(1+2 a m^{2}\right)\right| \geq v_{0} .
$$

Then, we have $2 m u_{0}-v_{0}\left(1+2 a m^{2}\right) \geq v_{0}$ or $-2 m u_{0}+v_{0}$ $\left(1+2 a m^{2}\right) \geq v_{0}$. Thus, $m u_{0} \geq\left(a m^{2}+1\right) v_{0}$ or $m u_{0} \leq a v_{0} m^{2}$.

Case (i): $m u_{0} \geq\left(a m^{2}+1\right) v_{0}$. 


$$
\begin{aligned}
u_{0}^{2}-d v_{0}^{2} & =-p, \\
\left(m u_{0}\right)^{2}-d\left(m v_{0}\right)^{2} & =-p m^{2}, \\
\left(\left(a m^{2}+1\right) v_{0}\right)^{2}-d\left(m v_{0}\right)^{2} & \leq-p m^{2}, \\
v_{0}^{2} a^{2} m^{4}+v_{0}^{2}+2 a m^{2} v_{0}^{2}-v_{0}^{2} a^{2} m^{4}-a m^{2} v_{0}^{2} & \leq-p m^{2}, \\
v_{0}^{2}+a m^{2} v_{0}^{2} & \leq-p m^{2} .
\end{aligned}
$$

Case (ii): $m u_{0} \leq a v_{0} m^{2}$

$$
\begin{aligned}
u_{0}^{2}-d v_{0}^{2} & =-p \\
\left(m u_{0}\right)^{2}-d\left(m v_{0}\right)^{2} & =-p m^{2} \\
\left(a v_{0} m^{2}\right)^{2}-d\left(m v_{0}\right)^{2} & \geq-p m^{2} \\
-a m^{2} v_{0}^{2} & \geq-p m^{2} \Longrightarrow p \geq a v_{0}^{2} \geq a
\end{aligned}
$$

In both cases, we find a contradiction.

Theorem 2. Let $\Delta \in \mathbb{N}$ be a fundamental discriminant, $\Delta=4 d$ or $\Delta=d \equiv 1(\bmod 8), d=(a m)^{2}+a$, where $a, m \in \mathbb{N}, m \geq 1, a>2$ and $a$ is not a prime number, then $h(d)>1$

Proof. Let $p$ be a prime number and $p \mid a$; hence, $a=e p$, where $e$ is square-free integer, which implies $d=\mathrm{pr}$ for $r=p e^{2} m^{2}+e$.

From Theorem 1 and Proposition 1, we obtain

Case (i): $\langle p, \sqrt{d}\rangle$ is not a principal ideal of $\mathcal{O}_{k}$ if $\Delta=4 d$ Case (ii): $\langle p,(p+\sqrt{d}) / 2\rangle$ is not a principal ideal of $\mathcal{O}_{k}$ if $\Delta=d \equiv 1(\bmod 8)$

In both cases, we find that $h(d)>1$.

The following proposition gives us a condition for solving the equation $x^{2}-\mathrm{d} y^{2}=c a, c \in\{-1,1\}$

Proposition 2. Let $d=a b$, where $a, b \in \mathbb{N}$, be a positive integer that is not a perfect square and suppose that $a$ is square-free and $a<b, c \in\{-1,1\}$. If the equation $x^{2}-d y^{2}=$ ca is solvable, then $\ell=\ell(\sqrt{d}) \equiv 2^{c+2}(\bmod 4)$.

Proof. By [16] (Proposition 3.1), it follows that $\ell=\ell(\sqrt{d})$ is even and $Q_{(\ell / 2)}=a$. We put $k=(\ell / 2)$ in (5). We obtain

$$
A_{(\ell / 2)-1}^{2}-\mathrm{d} B_{(\ell / 2)-1}^{2}=(-1)^{(\ell / 2)} a .
$$

Hence, $(-1)^{(\ell / 2)}=c$. We get

$$
\frac{\ell}{2}=\left\{\begin{array}{l}
2 r, \quad \text { if } c=1 \\
2 r+1, \quad \text { if } c=-1
\end{array}\right.
$$

So,

$$
\ell \equiv \begin{cases}0(\bmod 4), & \text { if } c=1 \\ 2(\bmod 4), & \text { if } c=-1\end{cases}
$$

This completes the proof.
Example 5. Let $d=46=2 \times 23=a b$ since

$$
\sqrt{46}=\langle 6 ; \overline{1,3,1,1,2,6,2,1,1,3,1,12}\rangle .
$$

Hence, $\ell=\ell(\sqrt{46})=12$, and the equation $x^{2}-46 y^{2}=$ -2 has no integer solutions.

Example 6. Let $d=11046=21 \times 526=a b$ since

$$
\sqrt{11046}=\langle 105 ; \overline{10,210}\rangle .
$$

Hence, $\ell=\ell(\sqrt{11046})=2$, and the equation $x^{2}-$ $11046 y^{2}=21$ has no integer solutions.

We use Theorem 1 and Proposition 2 in order to prove the following theorem, which gives a sufficient condition for $h(d)>1$ in two cases.

Theorem 3. Let $\Delta=4 d \in \mathbb{N}$ be a fundamental discriminant and suppose that $c=1$ if $d \equiv 2(\bmod 4)$, and $c=2$, if $d \equiv$ $3(\bmod 4) ; d=a b$, where $a, b \in \mathbb{N}, a<b$ and $a \equiv 3(\bmod 4)$, $\ell(\sqrt{d}) \equiv 2^{c}(\bmod 4)$. Then,

(a) If $c=1$ and $8+(a+1)$ then $h(d)>1$

(b) If $c=2$ then $h(d)>1$

Proof

(a) From Proposition 2, the equation $x^{2}-\mathrm{d} y^{2}=a$ is unsolvable. Since $a \equiv 3(\bmod 4)$ and $8+(a+1)$, then the equation $x^{2}-\mathrm{d} y^{2}=-a$ is unsolvable. By Theorem 1 , it follows that $\langle a, \sqrt{d}\rangle$ is not a principal ideal; then, $h(d)>1$.

(b) From Proposition 2, the equation $x^{2}-\mathrm{d} y^{2}=-a$ is unsolvable. Since $a \equiv 3(\bmod 4)$, then the equation $x^{2}-\mathrm{d} y^{2}=a$ is unsolvable. By Theorem 1 , it follows that $\langle a, \sqrt{d}\rangle$ is not a principal ideal; then, $h(d)>1$

Example 7. Let $\Delta=44184=4 d ; d=11046=3 \times 3682=$ $a b \equiv 2(\bmod 4), a \equiv 3(\bmod 4), 8+(a+1)$, and $\ell(\sqrt{d})=2$. Then, $h(d)>1$.

Example 8. Let $\Delta=7564=4 d ; d=1891=31 \times 61=a b \equiv$ $3(\bmod 4), a \equiv 3(\bmod 4)$, and $\ell(\sqrt{d})=36$. Then, $h(d)>1$.

\section{Data Availability}

No data were used to support this study.

\section{Conflicts of Interest}

The authors declare that they have no conflicts of interest.

\section{Acknowledgments}

This work was supported by Tishreen University. 


\section{References}

[1] C. F. Gauss, Disquisitiones Arithmeticae, Springer-Verlag, Berlin, Germany, 1986.

[2] A. Biró, "Yokoi's conjecture," Acta Arithmetica, vol. 106, no. 1, pp. 85-104, 2003.

[3] A. Biró, "Chowla's conjecture," Acta Arithmetica, vol. 107, no. 2, pp. 179-194, 2003.

[4] R. A. Mollin, "On the insolubility of a class of diophantine equations and the nontriviality of the class numbers of related real quadratic fields of richaud-degert type," Nagoya Mathematical Journal, vol. 105, pp. 39-47, 1987.

[5] D. Byeon, M. Kim, and J. Lee, "Mollin's conjecture," Acta Arithmetica, vol. 126, no. 2, pp. 99-114, 2007.

[6] A. Biro' and K. Lapkova, "The class number one problem for the real quadratic fields $\mathbb{Q}\left(\sqrt{(a n)^{2}+4 a}\right)$," Acta Arithmetica, vol. 172, no. 2, pp. 117-131, 2016.

[7] D. Byeon and J. Lee, "A complete determination of Rabinowitsch polynomials," Journal of Number Theory, vol. 131, no. 8, pp. 1513-1529, 2011.

[8] D. Byeon and H. K. Kim, "Class number 1 criteria for real quadratic fields of richaud-degert type," Journal of Number Theory, vol. 57, no. 2, pp. 328-339, 1996.

[9] D. Byeon and H. K. Kim, "Class number 2 criteria for real quadratic fields of Richaud Degert type," Journal of Number Theory, vol. 62, pp. 257-272, 1997.

[10] A. Hoque and K. Chakraborty, "Pell-type equations and class number of the maximal real subfield of a cyclotomic field," The Ramanujan Journal, vol. 46, no. 3, pp. 727-742, 2018.

[11] A. Hoque and H. K. Saikia, "On the class-number of the maximal real subfield of a cyclotomic field," Quaestiones Mathematicae, vol. 39, no. 7, pp. 889-894, 2016.

[12] K. Lapkova, "Class number one problem for real quadratic fields of a certain type," Acta Arithmetica, vol. 153, no. 3, pp. 281-298, 2012.

[13] R. A. Mollin, "Lower bounds for class numbers of real quadratic and biquadratic fields," Proceedings of the American Mathematical Society, vol. 101, no. 3, 1987.

[14] H. Sankari and A. Issa, "Lower bound for the class number of $\mathbb{Q} \sqrt{n^{2}+4}, "$ International Journal of Mathematics and Mathematical Sciences, vol. 2020, Article ID 9519613, 4 pages, 2020.

[15] F. Halter-Koch, Quadratic Irrationals: An Introduction to Classical Number Theory, CRC Press, Boca Raton, FL, USA, 2013.

[16] R. A. Mollin and A. Srinivasan, "Pell equations: non-principal Lagrange Criteria and central norms," Canadian Mathematical Bulletin, vol. 55, no. 4, pp. 774-782, 2012.

[17] S. Alaca and K. S. Williams, Introductory Algebraic Number Theory, Cambridge University Press, Cambridge, England, 2004. 